\title{
Ferromagnetic inclusions in silicate thin films: insights into the magnetic properties of cosmic grains
}

\author{
Z. Djouadi ${ }^{1}$, J. Gattacceca ${ }^{2}$, L. d'Hendecourt ${ }^{1}$, P. Rochette ${ }^{2}$, A. P. Jones ${ }^{1}$, C. Davoisne ${ }^{3}$, H. Leroux ${ }^{3}$, and J. Borg ${ }^{1}$ \\ 1 Institut d'Astrophysique Spatiale (IAS), Bâtiment 121, Université Paris-Sud 11 and CNRS (UMR 8617), 91405 Orsay, France \\ e-mail: zahia.djouadi@ias.u-psud.fr \\ 2 CEREGE, Université Aix-Marseille 3 and CNRS, BP 80, 13545 Aix-en-Provence Cedex 4, France \\ ${ }^{3}$ Laboratoire de Structure et des Propriétés de l'Etat Solide, UMR 8008, Université des Sciences et Technologies de Lille, \\ Bâtiment C6, 59655 Villeneuve d'Ascq Cedex, France
}

Received 16 March 2007 / Accepted 11 April 2007

\begin{abstract}
Context. We recently reported the formation of metallic inclusions in an amorphous and/or crystalline silicate matrix by thermal annealing of thin films in reducing atmospheres. Experimentally, the obtained microstructures closely resemble those of the glass with embedded metal and sulphides (GEMS) found in chondritic porous interplanetary dust particles (CP IDPs). We present here the magnetic properties of these synthetic samples.

Aims. In this paper we report the detection and measurements of single domain and super paramagnetic ferromagnetic inclusions (SD/SP) in annealed silicate thin films of composition analogous to interstellar silicates and discuss the implications for the alignment of cosmic grains in astrophysical environments, in the presence of weak magnetic fields.

Methods. We investigate the magnetic properties of synthesized laboratory silicate samples by measuring their magnetization when subjected to a given magnetic field. The measurements were performed at different temperatures including those compatible with interstellar dust.

Results. The high values of remanent magnetization at saturation obtained in this work suggest the ability of our samples to indefinitely maintain a significant magnetization which may contribute to their alignment in weak magnetic fields.

Conclusions. From our laboratory experimental simulation we propose that interstellar grains contain iron in form of nm-sized metallic beads. This can explain the non-detection of iron in interstellar grains. These inclusions could play a role in the alignment of grains. We propose a possible scenario for the magnetization of the cosmic grains and give a minimum value for the magnetic susceptibilty for GEMS.
\end{abstract}

Key words. ISM: dust, extinction - ISM: magnetic fields - galaxies: magnetic fields

\section{Introduction}

In order to explain the polarization of starlight reflected by dust in interstellar clouds, as noted long ago (Hiltner 1949; Hall 1949), aspherical dust grains aligned to some degree by the interstellar magnetic field need to be invoked. These spinning grains obscure the starlight and their alignment is believed to result from their rotational dynamics within the ambient magnetic field. Historically, grain alignment was first studied in 1951 by Davis \& Greenstein (hereafter DG) but they failed to explain the persistence of the alignment in an environment where the grain is subject to the random effects of collisions between grains and the surrounding gas leading to the loss of the alignment, especially in environments where the magnetic field is weak (about $5 \mu \mathrm{G}$ for the interstellar medium, Heiles 1996). Jones \& Spitzer in 1967 reconsidered the DG model and proposed that the only way to explain the alignment of interstellar grains by magnetic fields as low as $1 \mu \mathrm{G}$, was to assume that iron atoms are present in the grains either as small clusters of about 100 atoms each, the grain is thus called superparamagnetic (SP hereafter) characterized by a large paramagnetic susceptibility or as ferromagnetic inclusions. The value of the magnetic field required for grain orientation with ferromagnetic and superparamagnetic grains is estimated to be between $10^{-2}$ and $5 \mu \mathrm{G}$
(Jones \& Spitzer 1967, and references therein). Duley (1978) discussed the ability of the core-mantle grains to align in a magnetic field; he calculated the magnetic fields required for the cases of a magnetic core-paramagnetic mantle and super paramagnetic core-paramagnetic mantle. Duley predicted that $0.1-$ $0.3 \mu \mathrm{m}$ in radius grains with icy mantles on $\mathrm{MgO} / \mathrm{FeO} / \mathrm{SiO}$ cores can be aligned in magnetic fields of about $1 \mu \mathrm{G}$. He also predicted that small $\mathrm{MgO} / \mathrm{FeO} / \mathrm{SiO}$ grains should be aligned in regions where conditions prohibit the growth of ice. Mathis proposed a model to explain the alignment of interstellar grains in the galactic magnetic field (Mathis 1986). He assumed that interstellar grains are composed of tiny individual grains some of them comprising superparamagnetic material (e.g. magnetite or iron). These particles have a very large paramagnetic susceptibility and would provide a promising alignment mechanism as suggested by Jones \& Spitzer. A silicate grain was assumed to be aligned in the galactic magnetic field if, and only if, it contains at least one SP particle (Mathis 1986). As proposed by Martin (1995), the GEMS (Glass Embedded with Metal and Sulphides) found in some chondritic porous interplanetary dust particles (CP IDPs) could be a simple solution to the mystery of interstellar grain alignment.

Moreover, starlight polarization can also be used as a probe to determine the magnetic field structure prevailing in a given 
environment. In this context Lazarian (2003) devoted his review article to the investigation of the magnetic fields in different astrophysical environments via the measurement of the polarimetry of the scattered light.

Despite the variety of theoretical and observational studies devoted to explain and understand the grain alignment, this problem, now six decades old, still remains to be resolved and no consensus has yet emerged to explain the grain properties in all astrophysical environments.

Finally, no experimental study has yet been devoted to the determination of magnetic properties of extraterrestrial samples and their analogues. In this paper we investigate the consequences of the Fe-Ni metallic inclusions produced in material analogous to the IDPs produced in the laboratory (Davoisne et al. 2006). We determine the magnetic properties of these samples and discuss the implications for the alignment of cosmic grains.

\section{Experiments}

As described in our previous papers (Djouadi et al. 2005; Davoisne et al. 2006), our samples are thin amorphous silicate films, typically $100 \mathrm{~nm}$ thick, obtained by cold condensation of vapours onto $3 \mathrm{~mm}$ diameter diamond discs. This geometry ensures a high surface-to-volume ratio compatible with interstellar dust characteristics. The San Carlos olivine was used as the precursor, its composition is $\mathrm{Mg}_{1.8} \mathrm{Fe}_{0.19} \mathrm{Ni}_{0.01} \mathrm{SiO}_{4}$. Annealing experiments were performed in a tubular furnace under vacuum. Infrared spectroscopy is used to study the structural evolution after annealing (Djouadi et al. 2005). Some parts of the films are scraped off for transmission electron microscopy (TEM) analyses at the nanometer scale for structural and chemical characterization. Despite the low Fe and Ni abundance of the initial amorphous thin films, metallic precipitates are formed in the heated samples, they have been estimated to have sizes between 2 and $50 \mathrm{~nm}$ (Davoisne et al. 2006; Djouadi et al. 2006).

In this paper we focus on the magnetic properties of the annealed silicate films. We used different samples noted D1 and D2 (both heated at $1023 \mathrm{~K}$ ), ND2 (heated at $1273 \mathrm{~K}$ ) and two bare diamond discs, used as blanks.

Magnetic measurements, performed at CEREGE, Aix-enProvence, France, were conducted on the thin films to evaluate the magnetic properties of metal/silicate assemblages with an emphasis on the detection of super paramagnetic ferromagnetic particles.

The bulk magnetic properties of the thin films were evaluated by performing hysteresis cycles using a Micromag Alternating Gradient Force Magnetometer (AGFM) equipped with a cryostat. Magnetic moments can be measured with a sensitivity of $10^{-11} \mathrm{~A} \mathrm{~m}^{2}$ at temperatures ranging from $10 \mathrm{~K}$ to $300 \mathrm{~K}$ and in magnetic fields up to $1 \mathrm{~T}$ (corresponding to $10^{10} \mu \mathrm{G}$ ). A detailed description of an AGFM is given by Flanders (1988).

The hysteresis cycles provide the following magnetic parameters: coercivity $\left(B_{\mathrm{c}}\right)$, saturation magnetization $\left(M_{\mathrm{s}}\right)$, remanent magnetization at saturation $\left(M_{\mathrm{rs}}\right)$ as well as high-field (paramagnetic + diamagnetic) susceptibility. The coercivity of remanence $\left(B_{\text {cr }}\right)$ can also be measured by applying back-fields on a previously saturated sample and measuring in a zero magnetic field. By saturating magnetically the sample at $10 \mathrm{~K}$ and having it warmed in zero magnetic field, it is also possible to evaluate the unblocking temperature spectrum of the remanent magnetization. This is a key aspect in the detection of superparamagnetic iron grains.

We also measured the remanent magnetization at saturation $\left(M_{\mathrm{rs}}\right)$ for each sample with a $2 \mathrm{G}$ DC SQUID

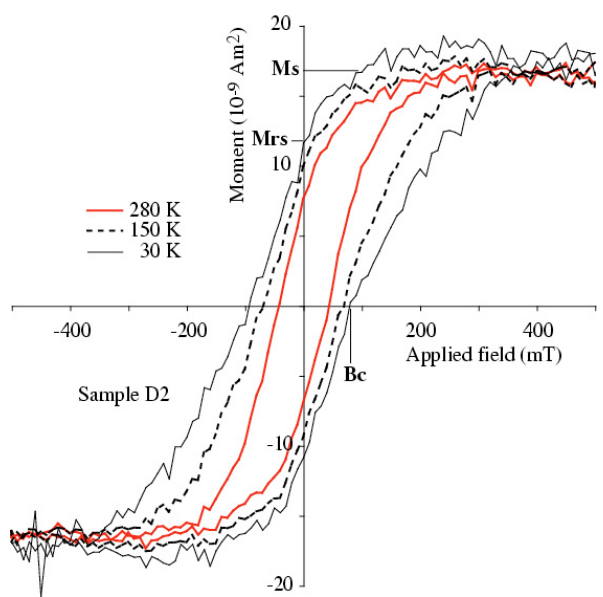

Fig. 1. Hysteresis cycles for sample D2 (room temperature $280 \mathrm{~K}, 150$ and $30 \mathrm{~K}$ ). The cycles are corrected for the high magnetic field susceptibility to show only the ferromagnetic signal (see text).

cryogenic magnetometer (sensitivity $10^{-11} \mathrm{~A} \mathrm{~m}^{2}$ ) and normalized the AGFM moment measurements using this value. Indeed the AGFM intensity calibration appeared less precise than the $2 \mathrm{G}$ measurements.

\section{Results}

The hysteresis cycles performed on the two bare diamond substrates only show a diamagnetic signal due to the diamond itself, whereas the heated samples (D1, D2, ND2) show a ferromagnetic contribution superimposed on this diamagnetic signal. The three heated samples gave identical results and the figures provided in the following are representative of the three samples. Typical hysteresis cycles obtained at different temperatures for the thin films are given in Fig. 1. We also report in this figure the corresponding magnetic parameters $B_{\mathrm{c}}, M_{\mathrm{s}}$ and $M_{\mathrm{rs}}$. Each hysteresis cycle is corrected for the high field susceptibility in order to subtract the diamagnetic signal due to the diamond substrate and the paramagnetic and diamagnetic signals of the silicate in the thin film. After these corrections, only the ferromagnetic signal due to the sample remains and is visible in the hysteresis cycle.

The large $B_{\mathrm{cr}}$ values measured for the thin films, ranging from $80 \mathrm{mT}$ at room temperature to $200 \mathrm{mT}$ at $10 \mathrm{~K}$, indicate that metallic iron is the main ferromagnetic mineral in the sample rather than magnetite, in agreement with our previous study (Davoisne et al. 2006). The saturation magnetization $\left(M_{\mathrm{s}}\right)$ is proportional to the total metallic iron content $\left(218 \mathrm{~A} \mathrm{~m}^{2} \mathrm{~kg}^{-1}\right.$ for pure iron). For the sample data displayed in Fig. 1, the $M_{\mathrm{s}}$ value of $\sim 16.8 \times 10^{-9} \mathrm{~A} \mathrm{~m}^{2}$ indicates a metallic iron content of $\sim 77 \mathrm{ng}$ which is in good agreement with the total amount of iron present in a San Carlos olivine disc of $3 \mathrm{~mm}$ in diameter and $100 \mathrm{~nm}$ thick ( $93 \mathrm{ng})$. This indicates that most of the iron in the original olivine is transformed into metallic iron and that no significant fractionnation occured during the evaporation and deposition of the sample. From the hysterises cycles and using the magnetization curve slope around zero field, a rough estimate of the weak field reversible susceptibility $\chi_{0}$ can be derived: for our synthetic samples it ranges from $1.5 \times 10^{-13}$ to $2 \times 10^{-13} \mathrm{~m}^{3}$ between 30 and $280 \mathrm{~K}$.

The shape of the hysterisis cycle, in particular the $M_{\mathrm{rs}} / M_{\mathrm{s}}$ ratio, allows us to evaluate the domain state of the metallic grains. For equant grains like those observed in our samples, the 


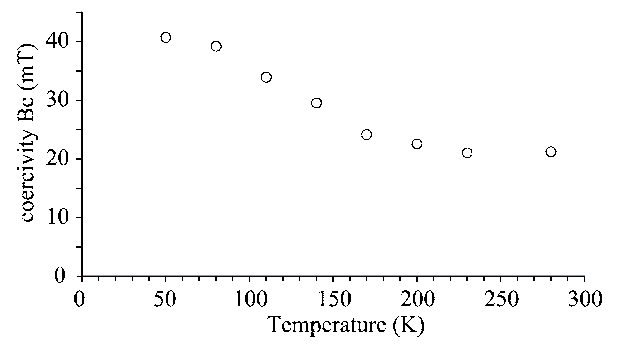

Fig. 2. Coercivity $\left(B_{\mathrm{c}}\right)$, given in $\mathrm{mT}$, plotted as a function of measurement temperature $(\mathrm{K})$ for the sample D1.

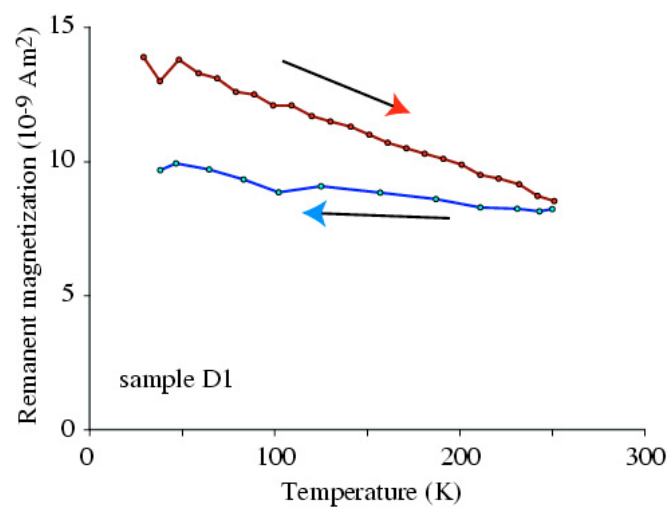

Fig. 3. Thermal demagnetization in the absence of magnetic field for the sample D1 from 30 to $250 \mathrm{~K}$ (upper curve). The initial saturation remanent magnetization was acquired at $10 \mathrm{~K}$ in a magnetic field of $1 \mathrm{~T}$. The demagnetization is followed by cooling between 250 and $30 \mathrm{~K}$ in the absence of a magnetic field (lower curve).

superparamagnetic (SP) to single domain (SD) transition occurs at around $25 \mathrm{~nm}$ at room temperature (Butler \& Banerjee 1975; Dunlop \& Özdemir 1997), while the SD to multidomain (MD) transition should be below $100 \mathrm{~nm}$. SD iron particles, that have cubic crystralline anisotropy with $\langle 100\rangle$ easy axis of magnetization, should have $M_{\mathrm{rs}} / M_{\mathrm{s}}=0.832$ (Dunlop \& Özdemir 1997). At low temperature the SP/SD threshold size will decrease as the cubic root of the temperature ratio (Dunlop \& Özdemir 1997), being about $8 \mathrm{~nm}$ at $10 \mathrm{~K}$.

Our hysteresis measurements at $10 \mathrm{~K}$ yield a $M_{\mathrm{rs}} / M_{\mathrm{s}}$ ratio up to 0.8 , indicating that most particles are in the SD state, i.e. larger than $8 \mathrm{~nm}$. Upon heating the $M_{\mathrm{rs}} / M_{\mathrm{s}}$ ratio of the samples decreases monotonically down to 0.45 , indicating that half of the grains that were $\mathrm{SD}$ at $10 \mathrm{~K}$ became $\mathrm{SP}$ at room temperature, i.e. that these grains are in the $8-25 \mathrm{~nm}$ size range. The absence of significant curvature of the cycle above $500 \mathrm{mT}$ indicates that the multidomain iron (above $\sim 50-80 \mathrm{~nm}$ ) is much less abundant, if present at all. The presence of SP grains is further demonstrated by the decrease in the coercivity $B_{\mathrm{c}}$ with temperature (Fig. 2).

Figure 3 displays the thermal demagnetization from $30 \mathrm{~K}$ to $250 \mathrm{~K}$ in a null magnetic field for a thin film previously saturated in a magnetic field of $1 \mathrm{~T}$ (at $10 \mathrm{~K}$ ). Part of the remanence $(\sim 40 \%)$ is lost at temperatures below room temperature, confirming the presence of a fraction (40 weight $\%$ maximum) of iron grains that are SP at room temperature, i.e. smaller than $25 \mathrm{~nm}$. The monotonic demagnetization indicates that grain size distribution spans the whole size range below $25 \mathrm{~nm}$ with no preferential clustering. All these inferences derived from magnetic measurements are in good agreement with the size range determined from TEM images for metallic inclusions in our samples (sizes 2 to $50 \mathrm{~nm}$ ).

\section{Discussion}

We report here magnetic measurements on synthetic samples of composition analogous to interstellar silicates, which contain metallic iron inclusions similar to those seen in the GEMS present in some CP IDPs. We show that our samples display superparamagnetic properties, suggesting that GEMS might also show similar behaviour. If GEMS indeed are interstellar dust grains (Bradley 1994, 1999), these SP inclusions would provide the necessary element for their alignment with the interstellar magnetic field (Jones \& Spitzer 1967). The bulk remanent magnetization that the ferromagnetic SD fraction may carry could be an additional component in the grain alignment. Two phenomena can be invoked to magnetize grains:

- cooling in a steady magnetic field and acquisition of a thermoremanent magnetization during grain formation;

- exposure to a magnetic field pulse (several mT) and thus acquisition of an isothermal remanent magnetization.

This second scenario would be much more efficient and may be more realistic as strong magnetic field pulses can be caused by lightning discharges in circumstellar clouds (Philipp et al. 1998). Another possibility would be an exposure to a magnetic field pulse in the proximity of a star and ejection of the magnetized grains in the interstellar medium. Magnetic field of tens of mT have been measured around some T-Tauri stars (Vallée 2003).

Most GEMS found in CP IDPs are 200 to $500 \mathrm{~nm}$ in diameter (Bradley 1995). For a density of about $3 \mathrm{~g} \mathrm{~cm}^{-3}$ the mass of the GEMS grains is in the range $10^{-14}$ to $10^{-13} \mathrm{~g}$. With a metallic iron content of $20 \%$ weight, these GEMS may have a saturation magnetization in the range $10^{-15}-10^{-14} \mathrm{~A} \mathrm{~m}^{2}$ depending on the size of the grain. Our data show that such grains can be magnetized up to $80 \%$ of their saturation magnetization if exposed to a strong magnetic field pulse at low temperature $(20 \mathrm{~K})$.

In modelling the behaviour of interstellar grains a pertinent parameter is the weak field reversible susceptibility $\chi_{0}$. For the above mentioned GEMS grains, our measurements on synthetic samples predict a $\chi_{0}$ in the range $10^{-14}-10^{-13} \mathrm{~cm}^{3}$. In theory this value can be extrapolated down to the very low interstellar magnetic fields (a few $\mu \mathrm{G}$ ). However, this only accounts for the magnetization rotation in the field. If the grains have a coherent remanence (as discussed above) and can rotate freely in the inducing field, a much higher effective susceptibility can be reached.

Recently Min et al. (2007) investigated the shape and composition of interstellar silicate grains. They based their study on a fit to the observed 10 and $20 \mu \mathrm{m}$ features and showed that interstellar silicate grains are highly magnesium rich $(\mathrm{Mg} /(\mathrm{Mg}+\mathrm{Fe})>$ $0.9)$. They also explain the unusual depletions of $\mathrm{Si}$ and $\mathrm{Mg}$ relative to Fe in lines of sight to the Small Magellanic Cloud (Weltey et al. 2001; Sofia et al. 2006) by the fact that the iron has to be in the form of refractory species such as metallic iron or iron oxide.

Since interstellar silicates are amorphous and $\mathrm{Mg}$-rich (Kemper et al. 2004, 2005; Min et al. 2007) and the silicate infrared absorption features are polarised (Dyck \& Beichman 1974) our work strongly supports the presence of metallic iron within the silicate matrix in dust. Additionally, the apparent lack of evidence for iron in the astronomical silicates, and the noted high iron depletion, support the inference that the iron is in a metallic form with no chemical bonds to other atoms that would be detectable by infrared spectroscopy. 


\section{Conclusion}

We investigated the magnetic properties of laboratory synthesized silicate samples containing metallic inclusions (resembling the GEMS in CP IDPs). We performed our measurements at temperatures down to the temperature of interstellar dust $(\sim 20 \mathrm{~K})$. We found that the magnetic properties of our samples are characterized by the presence of SD/SP metallic iron particles. At low temperatures the proportion of ferromagnetic metallic iron particles in the single domain state increases; these particles can be magnetized efficiently, either by exposure to a strong magnetic field pulse or by cooling in a steady ambient magnetic field. The magnetic properties derived would be consistent with the alignment of GEMS-like grains by interstellar magnetic fields. We propose a minimum value of the magnetic susceptibility for the GEMS grains comprised between $10^{-14}$ and $10^{-13} \mathrm{~cm}^{3}$ (this value does not take into account the possible free rotation of the grain under the magnetic field).

This work performed on synthetic samples may be applied in the future to natural grains, such as the CP IDPs containing GEMS. On January 15, 2006 the NASA Stardust mission delivered to Earth cometary samples, preliminary examinations showed that GEMS-like structures are widespread in the Wild2 grains (Keller et al. 2006; Zolensky et al. 2006). However, the crystallographic and composition analyses of these GEMS-like structures, revealed that they differ significantly from GEMS found in CP IDPs. It is proposed that these structures were produced in-situ during the deceleration of the Wild 2 grains in the aerogel collectors. The hot solar nebula origin for some of GEMS in IDPs is thus less probable (Chi et al. 2007).

In this work, we estimate for the saturation magnetization of GEMS found in IDPs to be around $10^{-15}-10^{-14} \mathrm{~A} \mathrm{~m}^{2}$. The magnetic measurements of these samples would require an instrumentation much more sensitive than the one used here (sensitivity of $10^{-11} \mathrm{~A} \mathrm{~m}^{2}$ ). The use of a submicron Hall probe $(\mathrm{Li}$ et al. 2002) would be a good candidate instrument for further analysis.
Acknowledgements. Z. Djouadi is grateful to the French INSU-CNRS program PNP for funding this work. She would also like to thank D. Deboffle for his invaluable help in the development of the experimental set-up and his assistance during the experiments.

\section{References}

Bradley, J. P. 1994, Science, 265, 925

Bradley, J. P. 1995, LPS, 26, 159

Bradley, J. P., Keller, L. P., Snow, T. P., et al. 1999, Science, 285, 1716

Butler, R. F., \& Banerjee, S. K. 1975, J. Geophys. Res., 80, 252

Chi, M., Ishii, H., Dai, Z. R., et al. 2007, LPS, 38, 2010

Davis, L. D., \& Greenstein, J. L. 1951, ApJ, 114, 206

Davoisne, C., Djouadi, Z., Leroux, H., et al. 2006, A\&A, 448, L1

Djouadi, Z., d'Hendecourt, L., Leroux, H., et al. 2005, A\&A, 440, 179

Djouadi, Z., Davoisne, C., d'Hendecourt, L., et al. 2006, SF2A-2006: Semaine de l'Astrophysique Francaise, 577

Duley, W. W. 1978, ApJ, 219, L129

Dunlop, D. J., \& Özdemir, Ö. 1997, Rock Magnetism, Fundamentals and frontiers (UK: Cambridge University Press), 573

Dyck, H. M., \& Beichman, C. A. 1974, ApJ, 194, 57

Flanders, P. J. 1988, J. App. Phys., 63, 3940

Hall, J. S. 1949, Science, 109, 166

Heiles, C. 1996, ASPC, 97, 457

Hiltner, W. A. 1949, Science, 109, 165

Jones, R. V., \& Spitzer, L. 1967, ApJ, 147, 943

Keller, L. P., Bajt, S., Baratta, G. A., et al. 2006, Science, 314, 1728

Kemper, F., Vriend, W. J., \& Tielens, A. G. G. M. 2004, ApJ, 609, 826

Kemper, F., Vriend, W. J., \& Tielens, A. G. G. M. 2005, ApJ, 633, 534 Lazarian, A. 2003, JQRST, 79, 881

Li, Y., Xiong, P., \& von Molnar, S. 2002, App. Phys. Lett., 80, 4644

Martin, P. G. 1995, ApJ, 445, L63

Mathis, J. S. 1986, ApJ, 308, 281

Min, M., Waters, L. B. F. M., de Koeter, A., et al. 2007, A\&A, 462, 667 Philipp, W., Hartquist, T. W., Mortfill, G. E., et al. 1998, A\&A, 331, 121 Sofia, U. J., Gordon, K. D., Clayton, G. C., et al. 2006, ApJ, 636, 753 Vallée, J. P. 2003, New Astron. Rev., 47, 85

Welty, D. E., Lauroesch, J. T., Blades, J. C., et al. 2001, ApJ, 554, L75

Zolensky, M. E., Zega, T. J., Yano, H., et al. 2006, Science, 314, 1735 